\title{
Reflets
}

Revue ontaroise d'intervention sociale et communautaire

\section{Enjeux de la nouvelle économie sociale dans la Péninsule acadienne}

\section{Isabelle Légère}

Volume 9, numéro 2, automne 2003

Travail et mieux-être

URI : https://id.erudit.org/iderudit/011096ar

DOI : https://doi.org/10.7202/011096ar

Aller au sommaire du numéro

Éditeur(s)

Reflets : Revue ontaroise d'intervention sociale et communautaire

ISSN

1203-4576 (imprimé)

1712-8498 (numérique)

Découvrir la revue

Citer cet article

Légère, I. (2003). Enjeux de la nouvelle économie sociale dans la Péninsule acadienne. Reflets, 9(2), 188-198. https://doi.org/10.7202/011096ar

Tous droits réservés (C) Reflets : Revue ontaroise d'intervention sociale et communautaire, 2002
Ce document est protégé par la loi sur le droit d'auteur. L'utilisation des services d'Érudit (y compris la reproduction) est assujettie à sa politique d'utilisation que vous pouvez consulter en ligne.

https://apropos.erudit.org/fr/usagers/politique-dutilisation/ 


\section{Enjeux de la nouvelle économie sociale dans la Péninsule acadienne}

Isabelle Légère, M.S.S.

Université d'Ottawa et doctorante en service social, Université Laval

\section{Introduction}

L'économie sociale est plus que jamais au cœur de l'actualité politique, économique et sociale au Canada. Dans le contexte actuel de l'appauvrissement des collectivités, l'économie sociale est considérée comme un moyen de repenser le rapport entre l'État, le marché et la communauté. Notre intérêt pour le développement économique communautaire a été suscité par la situation précaire dans laquelle vit une grande partie des habitantes et habitants dans la région de la Péninsule acadienne, alors que de plus en plus de familles acadiennes sont aux prises avec des problèmes de pauvreté. Les difficultés et les défis de la Péninsule acadienne soulèvent beaucoup de questions pour les leaders communautaires, notamment quant aux moyens à privilégier pour faire face aux problèmes.

L'objectif de cet article est d'explorer et de questionner la pertinence et l'utilité de l'économie sociale dans le contexte de communautés rurales éloignées et, plus spécifiquement, dans celui de la Péninsule acadienne. Ainsi, nous présenterons d'abord un profil de la situation de la Péninsule acadienne, pour ensuite situer certaines notions se dégageant des écrits sur la nouvelle économie 
sociale. Pour ce faire, nous nous appuierons sur certains élémentsclés que nous avons dégagés d'une analyse documentaire des écrits sur la nouvelle économie sociale, effectuée dans le cadre d'un mémoire de maitrise ${ }^{1}$. Finalement, nous nous demanderons si les approches de «Familles et Communautés d'abord» (FCD), un organisme qui cherche à favoriser l'émergence de projets d'économie sociale dans la Péninsule acadienne, correspondent aux éléments-clés de l'économie sociale.

\section{Contexte socio-économique de la Péninsule acadienne}

La Péninsule acadienne est un territoire d'environ $2000 \mathrm{~km}^{2}$, située à la limite nord-est du Nouveau-Brunswick, dans le comté de Gloucester. Baignant dans l'océan Atlantique, elle est bordée au nord par la baie des Chaleurs, à l'est par le golfe Saint-Laurent et elle est limitée à l'ouest par une frontière conventionnelle reliant la paroisse de New Bandon aux municipalités de Saint-Léolin, Paquetville, Saint-Isidore et Néguac. Cette région inclut trois pôles principaux qui structurent son territoire: Caraquet au nord, Tracadie-Sheila au sud et Shippagan à l'est. Dans l'ensemble, le territoire est très rural et la plus grande partie des structures administratives est située dans des districts de services locaux (DSL), c'est-à-dire des entités rurales non incorporées qui dépendent du gouvernement provincial. La Péninsule acadienne comprend près de 56180 habitantes et habitants, principalement francophones (95\%), qui vivent dans huit paroisses (habitations regroupées autour d'une église et plus petites qu'un village), dix villages et quatre villes. Ces appellations sont déterminées par la province, en fonction du nombre d'habitantes et d'habitants et de la superficie du territoire. La population de la Péninsule acadienne compose $7,7 \%$ de la population du NouveauBrunswick, qui comprend 729498 personnes (Statistique Canada 2003). La situation rurale de la Péninsule acadienne se caractérise 
par une «faible densité de population, [1'] absence de grandes métropoles, [1'] éloignement des marchés importants» (Polèse, Shermur et al. 2002; Statistique Canada 2003). Globalement, le sous-développement économique de la Péninsule acadienne est porteur de nombreux problèmes. Parmi eux, on trouve la perte d'emplois permanents ${ }^{2}$, le décrochage scolaire ${ }^{3}$, le taux élevé de suicides chez les jeunes de 20 à 24 ans $^{4}$, l'augmentation des familles monoparentales ${ }^{5}$, l'augmentation de la mendicité 6 et l'augmentation du nombre de foyers bénéficiaires des services de l'État (Statistique Canada 2003; DRHC 2003). Dans ce contexte de sous-développement, nombreux sont ceux qui font face à l'isolement social, la perte d'estime de soi et qui ont une immense difficulté à demeurer actifs.

Les personnes employées (77\% des gens de 15 ans ou plus) le sont dans des emplois saisonniers ou précaires, c'est-à-dire temporaires, surnuméraires, sur appel ou de remplacement, et seulement $23 \%$ d'entre elles travaillent à temps plein toute l'année (DRHC 2003). C'est donc dire que pendant la période creuse, de novembre à avril de chaque année, près de $65 \%$ de la population active reçoit des prestations d'assurance- emploi à condition, bien sûr, de répondre aux exigences requises (DRHC 2003; Johnson et McKee 1999). Peu importe la cause, nous constatons un chômage de «longue durée», c'est-à-dire qu'une travailleuse ou un travailleur est sans emploi depuis plus d'un an (Desfourny et al. 1998). Le revenu annuel total moyen (salaire, prestations d'aide au revenu, assurance-emploi, etc.) pour un individu est de $17000 \$$, alors que la moyenne provinciale est de 21000 \$ et la moyenne nationale de 25000 \$ (Statistique Canada 2003). De plus, une famille acadienne sur cinq est à faible revenu. Nous observons aussi que le pourcentage de familles à faible revenu a augmenté et que l'écart entre les familles à revenu élevé et celles à faible revenu s'élargit régulièrement (Gupta 2001).

De nos jours, la disponibilité des emplois continue d'être inférieure à la moyenne fédérale et provinciale, surtout dans le secteur des activités secondaires (transformations des produits) et tertiaires (services gouvernementaux). Globalement, l'activité socio-économique de la Péninsule repose surtout sur ses ressources 
naturelles (secteur d'activité primaire), comportant notamment la pêche, la cueillette de fruits sauvages et la tourbe. En effet, dans la Péninsule acadienne, l'économie est peu diversifiée et ne comporte aucune entreprise de grande taille, c'est-à-dire de plus de 300 employés. De plus, la qualité des emplois du secteur de la pêche s'est dégradée depuis les années 1980 en raison, d'une part, de la rareté du poisson et de la mise en place de quotas et, d'autre part, de la fin de l'aide gouvernementale pour l'emploi saisonnier. La diminution des prestations d'assurance-emploi depuis le milieu des années 1990 a mis fin à la pratique dite «10/42». Cette façon de faire était très courante à l'échelon local chez les employés saisonniers: un grand nombre de travailleuses et de travailleurs alternaient entre le travail et le chômage (dix semaines de travail permettaient de se qualifier pour 42 semaines d'assurance-chômage). Ces données montrent l'instabilité dans laquelle se retrouvent les gens de la Péninsule acadienne par rapport à leur emploi.

La Péninsule acadienne est composée d'une main-d'œuvre abondante, mais peu scolarisée: $37 \%$ des gens de plus de 15 ans détiennent un niveau de scolarisation inférieur à une neuvième année. Ces derniers travaillent surtout dans les industries primaires (activités économiques productrices de biens) (Beaudin 1999; Statistique Canada 2003). Cette situation s'explique par une double carence historique. D'une part, le manque d'institutions de formation a longtemps nécessité le départ des personnes souhaitant compléter des études postsecondaires et, d'autre part, le nombre restreint de postes à salaires compétitifs fait que les personnes qualifiées préferent aller vivre dans des régions où elles peuvent trouver des emplois mieux rémunérés.

Dans la Péninsule acadienne, la population a diminué de $5 \%$ entre 1996 et 2001 (par rapport à $1 \%$ au Nouveau-Brunswick) et on cherche à contrer l'exode des gens (Statistique Canada 2003). Les personnes partent à la recherche d'emplois permanents et stables ou afin de poursuivre une formation postsecondaire. Elles deviennent donc des travailleuses et des travailleurs plus qualifiés, mieux scolarisés et plus mobiles. En 2001, dans la Péninsule acadienne, l'émigration des jeunes de 15 à 24 ans était de 13,6\% et toutes les indications annoncent une augmentation massive en 
2003 (CBPA 2001). De plus, les résultats de l'étude Profil Jeunesse démontrent que $58 \%$ des jeunes de 15 à 24 ans ont quitté la Péninsule acadienne entre 1997 et 2001. Ajoutons maintenant que $70 \%$ des jeunes qui partent détiennent une formation postsecondaire (CBPA 2001). Ce problème d'émigration rurale est un enjeu important pour la survie et l'épanouissement de l'Acadie. «Sans emploi pour retenir ses jeunes dans ses collectivités, l'Acadie perd ainsi sa relève, ce qui constitue un désastre collectif», souligne Dallaire (1999: 30).

L'émigration des gens vers les grands centres urbains entraîne un manque alarmant de main-d'œuvre qualifiée dans la région. Si tous les jeunes adultes éduqués quittent la Péninsule acadienne, la région deviendra un endroit peuplé de personnes sans soutien social. Le vieillissement de la population acadienne et l'émigration des jeunes sont des phénomènes préoccupants qui entraînent une structure démographique et une main-d'œuvre vieillissantes (Beaudin 1999; Statistique Canada 2003).

\section{Table de travail sur l'économie sociale}

Dans le but de contrer les problèmes entourant la précarité et afin de créer des entreprises d'économie sociale, le projet pilote Familles et Communautés d'abord (FCD) a instauré, depuis juin 2000, une série de consultations sur le sujet et a mobilisé les leaders de la région. Sa vision est «que tous les citoyens et les citoyennes de la Péninsule acadienne se développent dans un cadre de santé globale et optimisent leur plein potentiel» (FCD 2000: 2). Les efforts de FCD ont permis la mise en place d'une vingtaine de Tables de travail qui regroupent plus de deux cents partenaires engagés dans des projets de développement social et communautaire.

Ces partenaires ont voulu établir un lien entre l'économie et le social, puisque l'un des buts de ce projet est de mobiliser les expertises. Une Table de concertation sur l'économie sociale a 
été mise en place pour identifier des sources de financement. Actuellement, les partenaires de la Table de concertation sur l'économie sociale, en collaboration avec les intervenantes et les intervenants de Familles et Communautés d'abord, travaillent à la mise en œuvre de leur plan d'action qui comprend une phase au cours de laquelle seraient développés les éléments suivants de références communes. Ceux-ci comprennent, premièrement, une étude d'impact social et économique du milieu associatif, communautaire et coopératif dans la Péninsule acadienne; deuxièmement, un exercice d'exploration des besoins et la mise en place des outils de développement pour l'émergence d'entreprises d'économie sociale dans la région et, finalement, la tenue d'un «Sommet sur l'économie sociale» prévu pour octobre 2004 (FCD 2003).

La Table de concertation sur l'économie sociale compte actuellement une quarantaine de partenaires qui se réunissent régulièrement. Ces partenaires clés proviennent de trois secteurs de l'économie: publique, sociale et privée. Ces exercices de consultation permettent la concertation des partenaires autour d'une table de travail et répondent à la mission de FCD. Les rencontres regroupent des leaders formels et informels de la Péninsule acadienne. La représentation des partenaires ne se fait pas par les décideurs (gouvernement), mais bien par le centre (leaders formels et informels) (FCD 2000).

\section{Définir l'économie sociale}

Si l'on retrouve, dans la littérature, un certain consensus autour de la définition de l'économie sociale, il n'existe aucune définition universelle de celle-ci. La définition proposée par le Groupe de Travail sur l'économie sociale cherche à préciser la signification et la portée de chacun des deux concepts: «économie» et «sociale». Le terme «économie» renvoie à la production concrète de biens et de services, ayant l'entreprise comme forme d'organisation et contribuant à une augmentation nette de la richesse collective. Le terme «sociale» réfere à la rentabilité sociale, et non purement 
économique de ces activités. Cette rentabilité s'évalue par la contribution au développement démocratique, par le soutien d'une citoyenneté active, par la promotion de valeurs et d'initiatives de prise en charge individuelle et collective (Groupe de Travail sur l'économie sociale 1996). Bref, les objectifs primordiaux de l'économie sociale sont d'adopter «un fonctionnement plus démocratique, des objectifs à la fois sociaux et économiques, qui produisent des biens et des services et qui sont une source d'emplois» (Welch 1999: 68).

\section{Analyse de l'approche du projet Familles et Communautés d'abord}

La première question qui a guidé notre analyse est de savoir comment FCD tient compte de l'empowerment individuel et collectif dans la mobilisation et la concertation des gens? Toute la dynamique de mobilisation et de concertation se définit autour d'un «espace d'absorption», où la règle du nombre, mais aussi de la diversité, permet la liberté de parole sur des enjeux fondamentaux, sans risque réel (Ninacs 2002). Selon cette optique, la Table de concertation sur l'économie sociale encourage les actrices et les acteurs partenaires à faire leur, le processus et les étapes à suivre et à en faire la promotion dans leurs milieux respectifs afin que les citoyennes et les citoyens se dotent de dispositifs permanents pour poursuivre le processus d'empowerment. De façon individuelle, les partenaires jouent un rôle dans le développement économique communautaire de la Péninsule acadienne en fonction de leurs propres choix ou forces (Parazelli et Tardif 1998). De cette façon, ils se donnent le contrôle pour solutionner leurs problèmes. Lorsque les partenaires, individuellement, se sont pris en main, ils peuvent travailler à une prise en charge de façon collective.

Cette prise en charge collective du développement cherche à intéresser les Acadiennes et les Acadiens à des projets économiques, sociaux et politiques en tant qu'actrices et acteurs de leur vie. La 
Table de concertation sur l'économie sociale, par la concertation du milieu, vise la prise en charge collective et le contrôle de ses ressources afin d'établir un partage équitable des moyens pour aboutir à une justice sociale. La Table de concertation sur l'économie sociale répond donc aux principes de l'empowerment individuel et collectif dont l'un des principaux objectifs est d'offrir à la communauté les outils nécessaires pour s'auto-analyser, se définir et résoudre ses propres problèmes (Eisen 1994).

Par ailleurs, est-ce que l'intervention communautaire faite par FCD répond aux principes de cette approche? Le projet FCD utilise l'approche de l'intervention communautaire, plus précisément celle du développement économique communautaire, en relation avec l'approche de l'économie sociale. Parmi ses objectifs, FCD vise la prise en charge (empowerment) individuelle et collective, le partenariat et la création d'emploi. Les approches en intervention communautaire sont des outils nécessaires pour répondre le mieux possible aux besoins des familles et des communautés d'une région désignée.

Selon Doucet et Favreau, l'intervention communautaire intervient à trois paliers: dans les communautés géographiques, d'intérêts et d'identités. Cependant, FCD intervient plutôt à deux paliers: dans les communautés géographiques et dans les communautés d'intérêts (Doucet et Favreau 1991; FCD 2000; DRHC 2003). Ces deux paliers permettent un travail étroit avec la communauté, car celle-ci est constituée, notamment, d'un «espace dans lequel des citoyens s'associent en raison de leurs intérêts individuels et collectifs» (FCD 2000: 9). Nous pouvons toutefois remarquer que même si FCD ne spécifie pas l'intervention dans les communautés d'identités, cette forme d'intervention semble intégrée dans la culture acadienne et dans celle de FCD, et ce, en fonction de l'appartenance culturelle que les résidantes et les résidants de la Péninsule acadienne partagent (Bagaoui et Dennie 1999).

En dernier lieu, est-ce que les objectifs de la Table de concertation sur l'économie sociale répondent aux principes et aux caractéristiques de l'emploi d'une entreprise d'économie sociale? Familles et Communautés vise d'abord, par l'empowerment 
et le partenariat des membres de sa communauté, l'émergence d'entreprises d'économie sociale. En effet, les entreprises d'économie sociale peuvent offrir des emplois durables dans le secteur communautaire et en même temps, développer de nouveaux bassins d'emplois liés à l'émergence de différents besoins sociaux (Bergeron 2002). D'après Boucher et Favreau, la nouvelle économie sociale favorise les activités économiques à «utilité sociale», assurant en même temps leur «viabilité économique» (2000: 42). Adhérant à cette optique, FCD (2000) voit donc l'économie sociale comme une possibilité intéressante pour contrer les problèmes de pauvreté, d'exclusion, de chômage et de développement dans la Péninsule acadienne. Comme exemple d'entreprise d'économie sociale dans cette région, citons le cas du Centre de bénévolat de la Péninsule acadienne (CBPA) qui, en plus d'être une source d'emplois, est aussi «un lieu d'entraide, de travail social et de formation à l'emploi» (Favreau 1996:9). Le CBPA offre un exemple d'entreprise à finalité économique et sociale qui favorise la cohésion sociale. Les buts et les modalités du CBPA rejoignent les propos de Laville affirmant que l'importance de l'économie sociale réside dans la «création de vrais emplois professionnalisés», le "renforcement de la cohésion sociale par la mobilisation locale engendrée» et la "contribution à la citoyenneté par l'expression et l'action collective à propos de problèmes quotidiens» (1997: 112).

Par contre, comme le secteur de l'économie sociale comporte des limites en ce qui a trait la création d'emplois, que ce soit du point de vue du travail obligatoire ou de la situation des femmes, il sera important que FCD les prenne en considération dans son travail afin de faciliter l'émergence de nouvelles entreprises d'économie sociale dans la Péninsule acadienne. Ainsi, les actrices et les acteurs du milieu devront s'assurer que tous les besoins de la population sont pris en considération en matière de création d'emplois. De plus, il sera intéressant de s'inspirer d'expériences réelles en la matière, qu'elles proviennent du Québec ou d'autres provinces.

En conclusion, la Table de Concertation sur l'économie sociale cherche à répondre aux valeurs de l'économie sociale par son intervention. Elle permet de créer des espaces publics de consultation, de mobilisation et de concertation tout en laissant 
aux gens la liberté de s'exprimer à haute voix. De plus, elle permet aux partenaires de faire l'expérience du processus d'empowerment à partir de la réalité et des besoins du milieu, entraînant ainsi un déplacement radical du pouvoir (Kaye et Wolff 1998). En d'autres mots, la Table de concertation sur l'économie sociale vise une action axée sur les capacités et non sur les problèmes, une action centrée sur l'environnement et non sur les limites de l'individu.

Notre analyse nous amène donc à constater que dans l'ensemble, Familles et Communautés d'abord répond à la mission de l'économie sociale selon laquelle l'empowerment, la notion de communautaire et la création d'emploi sont essentiels au développement d'entreprises d'économie sociale. Bref, notre recherche valide le potentiel de développement d'entreprises d'économie sociale comme stratégie de survie pour la Péninsule acadienne.

\section{Bibliographie}

BAGAOUI, Rachid et Donald A. DENNIE (1999). «Le développement économique communautaire: nouveau départ pour le mouvement associatif franco-ontarien", Reflets, vol. 5, no 1, 75-94.

BEAUDIN, Maurice (1999). L'état des régions: La région économique du nord-est du Nouveau-Brunswick, Moncton, Institut de recherche sur le développement régional.

BERGERON, Gilles (2002). «Développement local en question», dans Marielle Tremblay, PierreAndré Tremblay et Suzanne Tremblay, éd., Développement local, économie sociale et démocratie, Québec, Presses de l'Université du Québec.

BOUCHER, Jacques et Louis FAVREAU (2000). «Développement local et économie sociale: nouveau défi du travail social», Cahiers de la Chaire de recherche en développement communautaire, série recherche \#2.

CENTRE DE BÉNÉVOLAT DE LA PÉNINSULE ACADIENNE - CBPA (2001). Profil Jeunesse de la Péninsule acadienne, Caraquet, CBPA, Université de Moncton, Campus de Shippagan.

DALLAIRE, Pierre (1999). Regard sur l'Acadie et ses rapports avec le Québec, Moncton, Les éditions d'Acadie.

DEFOURNY, Jacques, Louis FAVREAU et Jean-Louis LAVILLE (1998). Insertion et nouvelle économie sociale. Un bilan international, Paris, Socio-Économie Desclée de Brouwer.

DÉVELOPPEMENT DES RESSOURCES HUMAINES CANADA - DRHC (2003). Statistiques socio-géographiques, District Chaleur-Péninsule.

DOUCET, Laval et Louis FAVREAU (1991). Théorie et pratique en organisation communautaire, Québec, Presses de l'Université du Québec.

EISEN, A. (1994). «Survey of neighbourhood-based, comprehensive community empowerment initiatives», Health Education Quarterly, vol. 21, no 2, 235-252.

FAMILlES ET COMMUNAUTÉS D'ABORD (2003). Plan d'action révisé: Dossier de l'économie sociale, Caraquet, CBPA.

FAMILLES ET COMMUNAUTÉS D'ABORD (2000). Plan d'action quinquennal visant les familles 
et les communautés de la Péninsule acadienne, Caraquet, CBPA.

FAVREAU, Louis (1996). Mouvement sociaux, travail social et économie solidaire face à la crise de l'emploi et de l'État-providence, Chaire de Recherche en Développement Communautaire, Hull, UQAH.

GROUPE DE TRAVAIL SUR L'ÉCONOMIE SOCIALE (1996). Osons la solidarité, Rapport dans le cadre du Sommet sur l'économie et l'emploi, Québec.

GUPTA, Meenakshi (2001). «Ottawa@ Travail, Primes et licenciements: une histoire de deux phénomènes constatés dans la population active», Notre Capitale Sociale, vol. 1, no 1, 5-9.

JOHNSON, Marc et Isabelle McKEE-ALLAIN (1999). "La société et l'identité de l'Acadie contemporaine», dans Joseph Yvon Thériault, éd., Francophonies minoritaires au Canada L'état des lieux, Moncton, Les éditions de l'Acadie.

KAYE, Gillian et Tom WOLFF (1998). Concertation locale: Les clefs du succès, trad. de l'anglais par Sylvie Méthot, Québec, Réseau québécois de Villes et Villages en santé.

LAVILLE, Jean-Louis (1997). «Un nouveau contrat social», Possibles, vol. 21, no 2, 106-114.

NINACS, William A. (2002). «Le pouvoir dans la participation au développement local: dans un contexte de mondialisation", dans Marielle Tremblay, Pierre-André Tremblay et Suzanne Tremblay, éd., Développement local, économie sociale et démocratie, Québec, Presses de l'Université du Québec.

PARAZELLI Michel et Gilles TARDIF (1998). «Le mirage démocratique de l'économie sociale» dans Louise Boivin et Mark Fortier (éd.), L'économie sociale: l'avenir d'une illusion, Montréal, FIDES.

POLÈSE, Mario, Richard SHERMUR et al. (2002). La périphérie face à l'économie du savoir: La dynamique spatiale de l'économie canadienne et l'avenir des régions non métropolitaines du Québec et des provinces de l'Atlantique, Québec, Université du Québec, Institut national de la recherche scientifique.

STATISTIQUE Canada (2003). Données du recensement 2001, Ottawa, Gouvernement du Canada.

WELCH, David (1999). «L'économie sociale en Ontario français: analyse historique, pratiques actuelles et recherche de sens», Reflets, Revue ontaroise d'intervention sociale et communautaire, vol. 5, no 1, 54-74.

Note

1. L'article provient de mon mémoire de maitrise intitulé «La nouvelle économie sociale: une stratégie de survie pour la Péninsule acadienne?» déposé en août 2003 à l'École de service social de l'Université d'Ottawa.

2. Les emplois permanents sont remplacés par des emplois temporaires ou contractuels à salaire minimum.

3. Le décrochage scolaire est de $29 \%$ supérieur à la moyenne provinciale chez les élèves de 13 à 15 ans.

4. Le taux de suicide chez les jeunes de 20 à 24 ans est de $86 \%$ plus élevé que la moyenne canadienne.

5. L'augmentation des familles monoparentales est de $19 \%$ par année.

6. L'augmentation de la mendicité est de $65 \%$ par année. 